\title{
Immediate effects after stochastic resonance whole-body vibration on physical performance on frail elderly for skilling-up training: a blind cross-over randomised pilot study
}

\author{
Slavko Rogan • Dietmar Schmidtbleicher • \\ Lorenz Radlinger
}

Received: 25 December 2013/Accepted: 28 February 2014/Published online: 4 April 2014

(C) Springer International Publishing Switzerland 2014

\begin{abstract}
Objective This pilot study examined the feasibility outcome recruitment, safety and compliance of the investigation for stochastic resonance whole-body vibration (SRWBV) training. Another aim was to evaluate the effect size of one SR-WBV intervention session on Short Physical Performance Battery (SPPB), Expanded Timed Get Upand-Go (ETGUG), isometric maximal voluntary contraction (IMVC) and rate of force development (IRFD) and chair rising (CR).

Design Randomised double-blinded controlled crossover pilot study.

Method Feasibility outcomes included recruitment, safety and compliance. For secondary outcomes, SPPB, ETGUG, IMVC, IRFD and CR were measured before and 2-min after intervention. Nonparametric Rank-Order Tests of Puri and Sen L Statistics to Ranked Data were proposed. Wilcoxon signed-ranked tests were used to analyse the differences after SR-WBV intervention and sham intervention. Treatment effects between the interventions were compared by a Mann-Whitney $U$ test.

Results Among 24 eligible frail elderly, 12 agreed to participate and 3 drop out. The adherence was 15 of 24 intervention sessions. For secondary outcome, effect sizes
\end{abstract}

(Clinical trial register: NCT01543243).

S. Rogan $(\bowtie) \cdot$ L. Radlinger

Bern University of Applied Sciences, Discipline Physiotherapy,

Murtenstrasse 10, 3008 Bern, Switzerland

e-mail: slavko.rogan@bfh.ch

D. Schmidtbleicher

Institute of Sport Sciences, Goethe-University, Frankfurt,

Germany
(ES) for SR-WBV intervention on SPPB, ETGUG and CR were determined.

Conclusion This pilot study indicate that the training protocol used in this form for frail elderly individuals is feasible but with modification due to the fact that not all defined feasibility outcomes target was met. SR-WBV with $6 \mathrm{~Hz}$, noise level 4 shows benefit improvements on SPPB (ES 0.52), ETGUG (part sit-to-stand movement: ES 0.81; total time: ES 0.85) and CR (ES 0.66). Further research is desired to determine whether a new adapted training protocol is necessary for SR-WBV in the "skilling up" phase in frail elderly individuals.

Keywords Feasibility · Adherence · Attrition - Isometric maximal voluntary contraction - Isometric rate of force development

\section{Introduction}

Dynapenia describes age-associated loss of muscle strength, power and functional strength [1]. These losses affect negatively physical performance, which increases the risk of falling. Maintenance of physical performance into ageing is crucial to sustaining functional independence in normal everyday life [2].

Sensorimotor and strength training are valid intervention methods to increase strength, power and functional strength in the field of geriatrics. The question that arises what exercises are best suited to and most effective for elderly individuals who have marked physical limitations because of dynapenia and therefore want to start a training programme. Such populations are advised first to enter a "skilling up" before more traditional forms of training exercises are implemented [3]. What kinds of exercises are 
appropriate for "skilling up", however, needs to be answered [4]. Further, a classification of the current status of physical performance in elderly individuals for exercise training must be carried out. A classification assigns frail elderly individuals in skilling up and mobile and trained elderly individuals in conventional training [5].

One training method for skilling up could be wholebody vibration (WBV). Multiple available systematic reviews and meta-analyses have described positive effects after WBV training in elderly population [6-8]. There are two major types of WBV: sinusoidal (i.e. constant vibration frequency) (S-WBV) and stochastic resonance vibration (i.e. random vibration frequencies) (SR-WBV). During S-WBV the participants stand on a platform that vibrates vertically or side alternating [9] with a high frequency (20-50 Hz) over a small peak-to-peak amplitude (2-6 mm). For SR-WBV, the participants stand on two separate platforms that vibrate independently with frequencies between 1 and $12 \mathrm{~Hz}$ [10].

During WBV, mechanical vibration produces small changes in the muscle-tendon complex [11]. These changes activate the muscle spindles with stimulation of the Iaafferents, facilitating the alpha motor neuron output and contracting the extrafusal muscle fibres [12]. In contrast to S-WBV, stochastic signal components cause a simple collection and processing by the nervous system [13]. This occurs, because in relatively small nerve cell aggregations stochastic background activities are available. Furthermore, SR-WBV has the advantage that the nerve cells cannot adapt [14, 15]. The use of stochastic resonance vibration might be beneficial for frail elderly individuals, since the nervous systems ability to fully activate skeletal muscle voluntarily seems impaired in dynapenia, which might be activated through SR-WBV.

One pilot study applied SR-WBV to an intervention for untrained elderly individuals on postural control [16]. This study indicates that SR-WBV intervention is safe intervention method and untrained elderly rose faster from sitting to standing position. New treatments usually have to go through a series of test stages to determine whether they are feasible, safe and effective [17]. In this context it seems justified to perform a pilot study to assess the feasibility of applying SRWBV in frail elderly individuals. The findings of such a study can inform a larger scale main RCT study [17].

The aim of this pilot study was to perform a phase II trial to test immediate effects of SR-WBV on frail elderly individuals. Phase II trials are studies on safety and tolerability in the target population and first investigations study of effect and apportioning [17]. This pilot study aimed (I) to evaluate the feasibility of the intervention process and the ability to recruit and retain frail elderly individuals for such an intervention, and (II) to evaluate the immediate effects of one session SR-WBV intervention on physical performance, gait, isometric maximal voluntary contraction (IMVC), isometric rate of force development (IRFD) and functional strength.

\section{Methods}

The design of this study was constructed following the statement of Thabane et al. [17]. This statement described how to report the results of pilot investigations.

\section{Design}

This study involved a randomised cross-over design with blinding of the participants and blinding of the assessors where nine participants completed a one session of SRWBV intervention and a one session of Sham intervention. A Zeptor med ${ }^{\circledR}$ vibration device (Frei Swiss AG, Zürich, Switzerland) was used for the application of a SR-WBV. Its footplates move three-dimensional up-down, forwardbackward and left-right. The participants stood with shoes in an upright position with slightly flexed hip, knee and ankle joints on the platform of the vibration device. SRWBV parameter for one session was: five repetitions of 1-min vibration and 1-min rest in between. The participants act as their control.

The participants were randomised to receive one session of SR-WBV intervention with a frequency at $6 \mathrm{~Hz}$, Noise level 4 in Period 1 and then a Sham intervention with a frequency at $1 \mathrm{~Hz}$, Noise level 1 (SR-WBV/Sham) in Period 2 after a wash-out phase or vice versa (Sham/SRWBV). The participants were tested before (pretest) and 2 min after intervention in period 1 (posttest) and again before starting period 2 (posttest) and $2 \mathrm{~min}$ after intervention in period 2 (pretest). Figure 1 shows the survey diagram.

\section{Participants}

Twelve frail elderly individuals (four male and eight female) living in a residential care setting participated in this study. Participant characteristics are given in Table 1. They were recruited by an oral presentation. In this pilot study, (pre-)frail was defined using the Physical Performance Classification for Elderly (PPCfE) proposed by Rogan et al. [18]. The PPCfE was determining by the total score of the short physical performance battery (SPPB). Status (pre-)frail: 0-6 points; status mobile: 7-10 points; status trained: $11-12$ points. (Pre-)Frail were defined if $\geq 6$ point was confirmed.

Participants were included if they were aged over 65 years, were able to stand without aids, were classified as Resident Assessment Instrument (RAI) performance level 


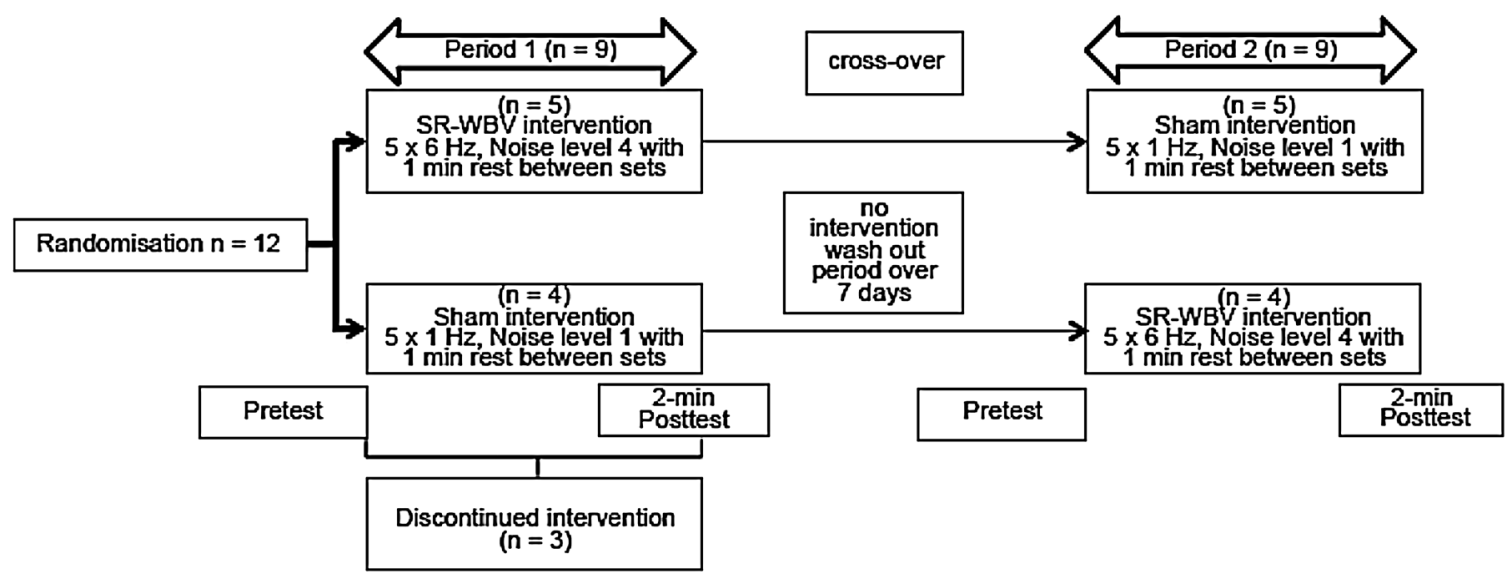

Fig. 1 Flow chart of the pilot study

Table 1 Summary description of the demographic variables at baseline of all recruited participants $(n=12)$ and analysed participants $(n=9)$ in means and SD

\begin{tabular}{lll}
\hline & $n=12$ & $n=9$ \\
\hline Age (years) & $86.3 \pm 6.9$ & $88.5 \pm 5.9$ \\
Height $(\mathrm{cm})$ & $166 \pm 0.1$ & $1.68 \pm 1$ \\
Weight $(\mathrm{kg})$ & $64.3 \pm 14.5$ & $68.8 \pm 14.3$ \\
Gender $(\mathrm{female} / \mathrm{male})$ & $8 / 4$ & $4 / 4$ \\
BMI $\left(\mathrm{Kg} / \mathrm{m}^{2}\right)$ & $23.2 . \pm 4.4$ & $28.3 \pm 4.7$ \\
Amount of individuals using & $10 \%$ (wheeled & $10 \%$ (wheeled \\
walking aids $(\%)$ & walker) & walker) \\
Presence of comorbidity: & & 2 \\
Hypertension & 2 & 3 \\
Diabetes & 3 & 1 \\
Coronary artery disease & 2 & 0 \\
Cerebrovascular disease & 1 & 1 \\
Asthma & 1 & 2 \\
Osteoporosis & 3 & 0 \\
Incontinence & 9 & \\
Cancer & 0 & \\
\hline
\end{tabular}

$B M I$ body mass index

$>0,{ }^{1}$ living in the Canton of Bern and had a score $>22$ in the Mini-Mental Status Examination. Exclusion criteria were: visual disturbances, lower or upper limb prostheses, acute joint diseases, acute thrombosis, acute fractures, acute infections, acute tissue damage, or acute surgical scars.

Randomisation

An independent research assistant performed the randomisation procedure with Microsoft Excel 2010. The participants were stratified by sex using a computer-

\footnotetext{
${ }^{1}$ RAI assess residents on admission to the nursing home, at least annually thereafter and on any significant change in status and to develop individualised, restorative plans of care.
}

generated sequence. Allocating occurred to either SRWBV/Sham or Sham/SR-WBV by means of sealed opaque envelopes that were distributed after the recruitment process. The study protocol has been approved by the Ethics Committee of the Canton of Bern (No. 142/11) and was registered under ClinicalTrial.gov: NCT01543243. All participants gave written informed consent.

Primary outcome: criteria of success

The criteria for success, important in a pilot study [17], were based on the primary feasibility objective and focused on recruitment, safety and compliance to the SR-WBV training. It was decided a priori to assess the feasibility with the following criteria for determining success: recruitment of a third of the residents that were eligible for the training session, a $15 \%$ attrition rate, and $80 \%$ adherence to the SR-WBV session were deemed acceptable. The recommended values are based from a previous pilot study with untrained elderly [16].

For safety, the authors interviewed the participants before and immediately after SR-WBV exercise on their well-being, feelings of stability and for adverse effects such as dizziness and pain during vibration. The responses for well-being and feeling of stability were: (1) worse, (2) the same, (3) better. Replied the participant with worse they were asked about dizziness or pain. These single questions were composed by the authors.

For attrition, we measured the number of participants lost at final follow-up.

For adherence to the intervention in total each participant trained over two units. An attendance list was kept to ensure enough training was completed.

\section{Secondary outcome}

Following measurements were intended from a previous pilot study with untrained elderly [19] and have been 
registered on ClinicalTrials.gov. Semi-Tandem stand (STS), SPPB, Expanded Timed Get Up-and-Go (ETGUG), single task (ST) and dual task (DT) conditions during walking, reaction time of the hand (RTH) and feet (RTF), isometric maximum voluntary force (IMVC) and isometric rate of force development (IRFD) and functional strength. The participants were familiarised with the SR-WBV intervention and measurements 1 week prior to data collection. During these measurements, it was found that frail elderly in contrast to untrained elderly not have the same physical performance level, to generate the same content and time required for an investigation or intervention. For this reason it was decided that out all measurements were carried out in this current pilot study. The outcome measurements have been carried out and will now be described in detail below.

The SPPB [20] assesses lower extremity function that allows prediction of the risk of physical damage.

It measures balance (semi-tandem stand, stand $=$ feet side by side and tandem stand), gait speed and lower limb strength during $5 \times$ chair rising (CR). The SPPB shows high reliability with an intraclass correlation coefficient (ICC) ranged from 0.88 to 0.92 [21].

Expanded Timed Get Up-and-Go (ETGUG) was used to measure dynamic balance. The ETGUG [22] has an ICC ranged from 0.55 to 0.97 and a test-retest reliability ranged from 0.54 to 0.85 . The participants had to perform a sit-tostand movement (SSM), to walk at their normal speed to the end of distance walkway, to turn around and to walk and sit back on the chair. At 2, 8 and $10 \mathrm{~m}$ along the walkway, markers were set (using coloured tape on the floor), allowing the measurement of the mean times after SS, between 0 and $2 \mathrm{~m}, 2-8 \mathrm{~m}$, turn, 12-18 $\mathrm{m}, 18-20 \mathrm{~m}$ and the total time (TT). Measurements were conducted using a digital hand stopwatch (Timex: Ironmen Triathlon, Middlebury, CL, USA). For the current study, the test was repeated twice with minimum 1-min rest in between.

A dynamometer measures IMVC and IRFD. Both were measured twice during each total of $5 \mathrm{~s}$ for leg extension. Between the measurements a break of $60 \mathrm{~s}$ was provided. An ICC for dynamometer measures was determined ranged from 0.95 to 0.98 for the lower extremity [23]. In this pilot study, the participants were sitting on a dynamometer chair at $90^{\circ}$ knee flexion. The participants responded to the instruction "3-2-1-go" as fast as possible and had to extend the leg isometrically as strong as possible for $5 \mathrm{~s}$. The analogue signal of the dynamometer was routed through an measurement amplifier (UMV, uk-labs, Kempen, Germany), digitised via a 12-bit A-/D- converter card (Meilhaus ME-2600i, SisNova Engineering, Zug, Switzerland) and analysed using Analog Digital Signal Processing software (ADS, uk-labs, Kempen, Germany). The IMVC was calculated by the highest strength value from the participant's individual force-time curves. The IRFD was extracted from the force-time calculating the steepness between the time points of $25 \%$ of IMVC and $75 \%$ IMVC [24].

For functional strength a CR test was used. The participants were instructed to sit on a 42-cm high chair and to place their arms across their chest. One completed CR was defined as moving from a starting seated position to standing fully upright and returning to the seated position. The participants responded to the instruction " $3-2-1-$ Go" to stand up and sit down five times as fast as possible. Timing began when the examiner said "Go" and stopped when the participant's buttocks touched the chair on the fifth repetition. Lord et al. [25] reported an ICC of 0.89 for reliability. The average of two trials was calculated.

\section{Statistical analyses}

Due to the low number of participants, nonparametric statistical procedures were carried out. The Nonparametric Rank-Order Tests of Puri and Sen L Statistics to Ranked Data were used for statistical analysis [26]. This statistical analysis includes following steps. First, the data distributions were changed to ranks. Second, carry-over effects between period one and period two (see Fig. 1) were determined using a two-factorial analysis of variance with repeated measures. If a carry-over effect was present, the data were analysed only with the first period data like a parallel design. If no carry-over effect was present, in a next step, data were analysed as SR-WBV intervention versus Sham intervention, irrespective of the order participants completed the training programmes using the Wilcoxon signed rank test and Mann-Whitney $U$ test [27].

According to Thabane et al. [17], in a pilot study, the focus is not primarily on the statistical analysis. This pilot study is in advance of a clinical intervention trial (RCT) which represents the validation phase and processing steps of the measurements and evaluation of intervention effect sizes (ES). Since this pilot study does not have the goal to identify significant intervention effects, but increasingly pays attention to evaluate ES on physical performance. For this reason no Bonferroni correction post hoc was performed. The level of significant was set at $\alpha<0.05$.

The ES were calculated for the differences within and between the groups and expressed as $r=\mathrm{Z} / \sqrt{ } \mathrm{N}$. For $\mathrm{r}$ an ES of 0.1 is considered a "small" effect, around 0.3 a "medium" effect and 0.5 and above, a "large" effect [28].

The programme $G^{*}$ Power 3 was used for post hoc analysis for power $1-\beta$ calculation [29]. All analyses were conducted using SPSS Version 20.0 (SPSS Inc., Chicago, IL, USA). 


\section{Results}

\section{Criteria of success}

The facility had a total of 100 residents from which staff representatives estimated 40 fulfilled eligibility criteria. One information session and one personal interview were held and visited by 40 residents. Due the lack of interest in advance, ten possible candidates were rejected. Six residents were excluded because physical testing was impossible. 24 residents were eligible to participate based on the inclusion-exclusion criteria and invited to participate. Of these, twelve frail elderly individuals consented to partake in the study. The other twelve participants indicated the following reasons for non-participation: no interest $(n=8)$, no motivation $(n=3)$ and personal reasons $(n=1)$. This resulted in a recruitment rate of $40 \%$ for the total sample frame. The inclusion rate-twelve invited to participate; twenty-eight enrolled-was $30 \%$.

The intended secondary outcome measurements could not be performed. STS, DT and ST and RT were removed from the secondary outcome measurement programme. The participants were not able to perform about $5 \mathrm{~s}$ the STS. For ST and DT, the participants did not have enough strength and endurance. The eyes of the participants were not always able to correctly detect the light stimulus for the $\mathrm{RTH}$ and RTF as well.

The attrition rate is $25 \%$ ( $n=3$ drop-outs). The reasons were a too high amount of measurements and too long measuring times $(n=1)$ and a lack of interest to continue in this study $(n=2)$. For adherence to the intervention, we had $72.7 \%$ (15 of 24 training sessions).

The participants were willing to be randomised. For safety, neither subjective nor objective side effects relating to the intervention used were reported.

Secondary outcome

Table 2 shows the intervention effect and ES after SRWBV training on the secondary outcomes between and within the SR-WBV intervention and sham intervention. Large ES after $6 \mathrm{~Hz}$, Noise 4 SR-WBV intervention on SPPB, ETGUG (SS and TT) and CR were determined. Sham intervention showed significant decreases on ETGUG between 0 and $2 \mathrm{~m}$, IMVC and IRFD of the right leg.

\section{Discussion}

The goal of this pilot study was (I) to evaluate the feasibility of the study process and the ability to recruit and retain frail elderly individuals for such an intervention, and (II) to assess the acute impact of one SR-WBV intervention session on physical performance, gait, IMVC, IRFD and functional strength.

The primary feasibility objectives provide the basis for interpreting the results of the pilot study and determining whether it is feasible to proceed to the next RCT study. This pilot study demonstrated that the planned study in this from is feasible but with modification due to the fact that not all scheduled and registered measurements could be realised, the attrition rate for frail elderly people is high and the adherence rate is low. Our aim to recruit 30 participants could not be reached. Those individuals that responded and visited an information and personal session showed no large inclusion rate. These findings indicate the importance of a new approach for recruitment of elderly individuals where questions and concerns about new interventions can be clarified.

\section{Criteria of success}

The strategy for the secondary outcome measurements was made in advance of this pilot study. As the study protocol was registered on ClinicalTrial.gov, the knowledge and experience was not given regarding selection and number of measurements on physical performance for frail elderly individuals. In old age, muscle strength and balance play an important role. These parameters are crucial to handle numerous situations of activity in daily life. On the other hand, frailty is the result of foregoing disease in multiple systems resulting in impaired muscle strength, postural control, endurance and mobility [30]. This means that frail elderly individuals are not resilient. Further, the vision in elderly individuals is reduced [31]. Originally, seven measurements for this study were provided with a scheduled time of one and a half hour. Subsequently, an intervention and then another measurement occur. For the frail elderly, this period is too long. Based on this finding, the originally intended measurements for the secondary outcome were reduced to SPPB, ETGUG and strength (IMVC, IRFD and functional strength). In this study, the selection of outcome measurements for frail elderly individuals was not suitable. In future studies, this point must be taken into account.

Compliance with the SR-WBV training and retesting was not good. Of the included frail elderly individuals, only nine completed the two training sessions. In addition, complete retest data were obtained from only nine frail elderly individuals. This is far more than the rate that could have been expected. It should be noted, however, that the mean compliance rate that was determined from one study mainly focused on immediate effects [19]. In contrast to this study, the pervious pilot study from Rogan et al. [19] showed a good compliance rate of $100 \%$. Another contrast is that the investigation was a randomised controlled study 
Table 2 Results for physical performance within and between SR-WBV and sham intervention (secondary outcomes)

\begin{tabular}{|c|c|c|c|c|c|}
\hline & $\begin{array}{l}\text { SR-WBV intervention } \\
(n=9) \text { Median (IQR) }\end{array}$ & $\begin{array}{l}P \text { within } \\
\text { ES (power) }\end{array}$ & $\begin{array}{l}\text { Sham intervention } \\
(n=9) \text { Median (IQR) }\end{array}$ & $\begin{array}{l}P \text { within ES } \\
\text { (power) }\end{array}$ & $\begin{array}{l}P \text { between intervention } \\
\text { (posttest) ES (power) }\end{array}$ \\
\hline SPPB (0-12 points) pretest & $7.0(4.5-9)$ & 0.121 & $7.0(6.0-8.5)$ & 0.564 & 0.730 \\
\hline 2-min posttest & $8.0(6.5-8.5)$ & $0.52(0.27)$ & $7.0(5.5-9.0)$ & $0.19(0.08)$ & $0.10(0.01)$ \\
\hline ETGUG SS, (s) pretest & $1.7(1.3-2.1)$ & $0.015^{\mathrm{a}}$ & $1.6(1.0-2.6)$ & 1.000 & 0.145 \\
\hline 2-min posttest & $1.0(0.9-1.9)$ & $0.81(0.54)$ & $1.8(1.0-1.9)$ & $0.00(0.00)$ & $0.06(0.01)$ \\
\hline ETGUG 0-2 m (s) Pretest & $3.4(2.5-4.7)$ & 0.173 & $3.4(1.8-3.9)$ & $0.017^{\mathrm{a}}$ & 0.791 \\
\hline 2-min posttest & $3.3(2.5-84.4)$ & $0.45(0.21)$ & $3.6(2.7-4.0)$ & $0.79(0.52)$ & $0.06(0.01)$ \\
\hline ETGUG 2-8 m, (s) pretest & $7.5(5.9-10.1)$ & 0.906 & $7.3(6.0-10.5)$ & 0.767 & 0.860 \\
\hline 2-min posttest & $7.2(6.1-10.0)$ & $0.04(0.005)$ & $7.2(6.3-8.8)$ & $0.10(0.06)$ & $0.04(0.01)$ \\
\hline ETGUG turn, (s) pretest & $8.2(6.1-10.8)$ & 0.314 & $7.9(6.1-9.5)$ & 0.086 & 0.659 \\
\hline 2-min posttest & $7.5(6.0-10.2)$ & $0.34(0.14)$ & $8.2(6.5-10.0)$ & $0.57(0.31)$ & $0.10(0.01)$ \\
\hline ETGUG $12-18 \mathrm{~m}$, (s) pretest & $7.2(6.3-10.3)$ & 0.314 & $7.3(6.5-10.3)$ & 0.314 & 0.860 \\
\hline 2-min posttest & $7.9(6.2-10.9)$ & $0.34(0.14)$ & $7.1(6.5-10.9)$ & $0.34(0.14)$ & $0.04(0.01)$ \\
\hline ETGUG $18-20 \mathrm{~m}$, (s) pretest & $6.7(5.2-8.5)$ & 0.080 & $6.3(4.7-8.2)$ & 0.051 & 0.500 \\
\hline 2-min posttest & $6.5(4.9-7.8)$ & $0.62(0.36)$ & $7.4(5.6-8.6)$ & $0.65(0.38)$ & $0.16(0.01)$ \\
\hline ETGUG TT, (s) pretest & $32.7(28.2-47.0)$ & $0.011^{\mathrm{a}}$ & $32.1(28.9-44.6)$ & 0.953 & 0.931 \\
\hline 2-min posttest & $29.7(24.3-42.8)$ & $0.85(0.58)$ & $31.0(27.6-42.3)$ & $0.02(0.05)$ & $0.02(0.01)$ \\
\hline IMVC left, (N) pretest & $266(205-502)$ & 0.779 & $291.8(222-455)$ & 0.767 & 0.423 \\
\hline 2-min posttest & $278(210-322)$ & $0.10(0.06)$ & $285.0(233-488)$ & $0.09(0.06)$ & $0.21(0.07)$ \\
\hline IMVC right, $(\mathrm{N})$ pretest & $274(195-504)$ & 0.176 & $322(254-490)$ & 0.594 & 0.837 \\
\hline 2-min posttest & $316(215-414)$ & $0.51(0.26)$ & $327(240-761)$ & $0.18(0.08)$ & $0.05(0.005)$ \\
\hline IRFD left, (N/s) pretest & $515(260-727)$ & 0.249 & $406(198-1,382)$ & 0.028 & 0.689 \\
\hline 2-min posttest & $437(118-1,937)$ & $0.47(0.23)$ & $327(240-761)$ & $0.18(0.08)$ & $0.12(0.006)$ \\
\hline IRFD right, (N/s) pretest & $389(262-869)$ & 0.753 & $482(423-736)$ & 0.694 & 0.535 \\
\hline 2-min posttest & $458(263-840)$ & $0.12(0.06)$ & $439(276-603)$ & $0.88(0.61)$ & $0.11(0.005)$ \\
\hline SSM, (s) pretest & $19.1(17.5-23.7)$ & 0.066 & $18.8(16.2-21.0)$ & 0.678 & 0.605 \\
\hline 2-min posttest & $18.0(15.1-22.2)$ & $0.61(0.35)$ & $19.9(16.2-22.5)$ & $0.14(0.07)$ & $0.13(0.006)$ \\
\hline
\end{tabular}

Median and interquartile range (IQR) values are pretest and 2-min posttest. $P$ values after pretest and 2-min posttest intervention were computed using Wilcoxon signed ranks test for within intervention and Mann-Whitney $U$ tests for between SR-WBV intervention and sham intervention SBBP Short Physical performance Battery, ETGUG Expanded Timed Get Up-and-Go, SS sit-to-stand movement, TT total time, IMVC isometric maximum voluntary contraction, IRFD isometric rate of force development, $S S M$ sit-to-stand movement, $E S$ effect size, $s$ seconds, $N$ Newton, $N /$ $s$ Newton/seconds

${ }^{\text {a }}$ Statistically significant difference $(p<.05)$ after pretest and posttest intervention

where the participants were measured pre- and post-interventional and trained once. Furthermore, data from the present study should be replicated in another study where the intervention is applied during several training sessions over several weeks. It can be expected that because of such a longitudinal design in an institutional setting the same less favourable compliance will occur. To maintain the compliance, motivation models should be used. With personal support, the motivation for SR-WBV training can be increased. Our findings warrant such follow-up studies based on these results.

Safety and acceptance are crucial features of the programme and therefore strict exclusion criteria were applied for this small cohort, particularly as it was a pilot study. Hopefully, the criteria can be released in time as experience is gained.
The duration of the wash-out period is crucial for a cross-over study. In this pilot study a seven-day wash-out period was chosen. This wash-out period extends through the absence of the carry-over effect, turns out to be suitable.

\section{Secondary outcome}

The Zeptor med ${ }^{\circledR}$ vibration device generates an unsynchronised multidimensional, low impact stochastic stimulus pattern superimposed on a sinusoidal basic activity [15]. In this way the participant is constantly kept off balance. The participants have to react as quickly as possible. The goal is to enhance body stability during the SRWBV session. Some studies described that SR-WBV modifies neuromuscular performance $[32,33]$. In this pilot 
study no improvements or tendencies towards improvements in any strength outcome parameters within a bout of five consecutive one-minute single SR-WBV sessions were determined.

These results are consistent with the study by Rogan et al. [19] but not with Schuhfried et al. [34]. The vibration intensity was adapted to the participant. They started with $3 \mathrm{~Hz}$ and increased the intensity until the limit of tolerance. The participants were able to stand without holding onto railings. A limitation in the present study was that the participants were holding onto the railings.

Apart from muscle strength, muscle power can be evaluated without using machines [35]. Muscle strength should be distinguished from muscle power. Strength is defined as the maximal force that a muscle can produce against a given resistance [1]. Muscle power is defined as the amount of force and speed generated [36]. Muscle power declines at a faster rate with increasing age than muscle strength [37, 38]. Yamashita et al. [39] described that improvement in muscle power, rather than muscle strength, can prevent falls. However, Knuttgen and Komi [40] described the assessment and quantification of physical performance as accomplished by use of the International System of Measurement (the SI) for force = Newton $(\mathrm{N})$ and power $=$ Watts $(\mathrm{W})$. The exercise intensity can therefore be quantified for power $=\mathrm{W}$ as energy expenditure or work performed per second or force times velocity. Muscle power is strongly correlated to performance of daily tasks [41, 42]. However, Iwamoto et al. [35] identify in their study not power, but functional strength. Functional strength of the lower extremity can be measured with SPPB (Part CR), ETGUG [part sit-to-stand (SS)] and CR [43]. It has been reported that the CR is associated with mobility [44]. Slow CR has been found to predict falls [45, 46] and hip fracture [47].

It may be possible that using SR-WBV training, sufficient stimuli can be generated to functional strength. During vibration, muscles adapt to length changes of the muscle and tendon complex, resulting in improved reflex activity [48]. In the current pilot study, large ES were determined after SR-WBV intervention on SPPB, ETGUG (SS, 12-18 $\mathrm{m}$ and TT) and CR.

In this study the participants are obese. The literature shows that adiposity contributes to frailty by reducing the ability of elderly individuals to perform daily live activities and increasing metabolic instability [49]. Obese, elderly individuals are particularly susceptible to sarcopenia. A combination of strength loss and fat gain are associated with decrease physical performance and disability, independent of morbidity [50, 51]. It is known that obese person negatively affect their adherence to exercise intervention [52]. This reflected with the attrition rate of this study.
In this current pilot study the participants shows (multi-) comorbidity conditions. All participants received medications because of comorbidity. In the management of comorbidity physical activity programmes can improve physical performance. The elderly with incontinence are not able to contract the pelvic floor muscle rapidly and strongly and treatment shows to be difficult. SR-WBV shows beneficial effects on rate of force development and elicits stretch reflexes in pelvic floor muscles [53]. A better continence status allows improving the mobility.

However, one limitation is that for SS during ETGUG and during CR many participants had to raise their arms to be able to manage this task.

Another possible explanation, and a limitation of the present pilot study, is that the strength test is only used for one muscle. For everyday life performance interaction of numerous muscles is required. In future studies more muscles should be measured on strength. Summarising the findings and limitations of this study, it becomes clear the study protocol must be reviewed again. Should the adapted protocol be successful, we envisage using SR-WBV as skilling-up exercise in frail elderly individuals. Prior to this, further formal evaluation of potential risks and outcomes is required in longitudinal studies.

The main limitation of this study is the small sample size that reduced the informative value of the described analysis. Further studies with lager groups are required.

A next step would be to replicate the new study protocol and findings in a new exercise group of frail elderly individuals.

A sample size calculation was conducted. The SPPB, for example, showed that the ES of the intervention was small at 0.10 . Based on the observed values for the SPBB, with $80 \%$ power at $\alpha$-level 0.05 with an ES 0.6 an estimated sample size of 411 participants for a 2-group RCT-design would be necessary.

Conflict of interest The authors report no conflict of interest

\section{References}

1. Clark BC, Manini TM (2008) Sarcopenia =/= dynapenia. J Gerontol Series A Biol Sci Med Sci 63(8):829-834

2. Reid KF, Naumova EN, Carabello RJ, Phillips EM, Fielding RA (2008) Lower extremity muscle mass predicts functional performance in mobility-limited elders. J Nutr Health Aging 12(7):493-498

3. Skelton DA, Dinan SM (1999) Exercise for falls management: rationale for an exercise programme aimed at reducing postural instability. Physiother Theory Pract 15(2):105-120

4. Landi F, Abbatecola AM, Provinciali M, Corsonello A, Bustacchini S, Manigrasso L, Cherubini A, Bernabei R, Lattanzio F (2010) Moving against frailty: does physical activity matter? Biogerontology 11(5):537-545. doi:10.1007/s10522-010-9296-1 
5. Rogan S, de Bruin ED (2013) Skilling-up exercise for deconditioned nursing home dwellers. Paper presented at the 18th annual ECSS Congress Barcelona, Barcelona

6. Marin PJ, Rhea MR (2010) Effects of vibration training on muscle power: a meta-analysis. J Strength Cond Res Natl Strength Cond Assoc 24(3):871-878. doi:10.1519/JSC. 0b013e3181c7c6f0

7. Marin PJ, Rhea MR (2010) Effects of vibration training on muscle strength: a meta-analysis. J Strength Cond Res Natl Strength Cond Assoc 24(2):548-556. doi:10.1519/JSC.0b013e3181c09d22

8. Rogan S, Hilfiker R, Herren K, Radlinger L, de Bruin ED (2011) Effects of whole-body vibration on postural control in elderly: a systematic review and meta-analysis. BMC Geriatr 11:72. doi:10. 1186/1471-2318-11-72

9. Cochrane DJ (2011) Vibration exercise: the potential benefits. Int J Sports Med 32(2):75-99. doi:10.1055/s-0030-1268010

10. Herren K, Rogan S, Hilfiker R, Radlinger L (2009) Vibrationen mit therapeutischen Effekten. Physioactive 44(5):39-44

11. Cochrane DJ, Loram ID, Stannard SR, Rittweger J (2009) Changes in joint angle, muscle-tendon complex length, muscle contractile tissue displacement, and modulation of EMG activity during acute whole-body vibration. Muscle Nerve 40(3):420-429. doi:10.1002/mus.21330

12. Roelants M, Delecluse C, Goris M, Verschueren S (2004) Effects of 24 weeks of whole body vibration training on body composition and muscle strength in untrained females. Int J Sports Med 25(1):1-5. doi:10.1055/s-2003-45238

13. Haas CT, Schmidtbleicher D (2006) Potential of Stochastic Resonance in Neurorehabilitation. Isokinet Exerc Sci 14(2):144-146

14. Haas CT, Turbanski S, Schmidtbleicher D (2007) Vibrationstraining in der Rehabilitation von Gang- und Gleichgewichtsstörungen. Focus Neurogeriatr 1(3):18-19

15. Haas CT, Turbanski S, Kaiser I, Schmidtbleicher D (2004) Biomechanical and physiological effects of oscillating mechanical stimuli in humans. Deutsche Zeitschrift für Sportmedizin 55(2):34-43

16. Rogan S, Hilfiker R, Schmid S, Radlinger L (2012) Stochastic resonance whole-body vibration training for chair rising performance on untrained elderly: a pilot study. Arch Gerontol Geriatr 55(2):468-473. doi:10.1016/j.archger.2012.02.011

17. Thabane L, Ma J, Chu R, Cheng J, Ismaila A, Rios LP, Robson R, Thabane M, Giangregorio L, Goldsmith CH (2010) A tutorial on pilot studies: the what, why and how. BMC Med Res Methodol 10:1. doi:10.1186/1471-2288-10-1

18. Rogan S, Radlinger L, Schmidtbleicher D, de Bie R, de Bruin ED (2012) Effect of whole-body vibration with stochastic resonance (SR-WBV) in an elderly population: impact on physical performance. Paper presented at the Ouvertüre Swiss Congress for Health Professions Winterthur, Switzerland

19. Rogan S, Radlinger L, Schmid S, Herren K, Hilfiker R, de Bruin E (2012) Skilling up for training: a feasibility study investigating acute effects of stochastic resonance whole-body vibration on postural control of older adults. Ageing Res 3(1):e5

20. Guralnik JM, Seeman TE, Tinetti ME, Nevitt MC, Berkman LF (1994) Validation and use of performance measures of functioning in a non-disabled older population: MacArthur studies of successful aging. Aging (Milano) 6(6):410-419

21. Ostir GV, Volpato S, Fried LP, Chaves P, Guralnik JM (2002) Reliability and sensitivity to change assessed for a summary measure of lower body function: results from the women's health and aging study. J Clin Epidemiol 55(9):916-921. doi:10.1016/ S0895-4356(02)00436-5

22. Botolfsen P, Helbostad JL, Moe-Nilssen R, Wall JC (2008) Reliability and concurrent validity of the expanded timed up-andgo test in older people with impaired mobility. Physiother Res Int 13(2):94-106. doi:10.1002/pri.394
23. Viitasalo JT, Salo A, Lahtinen J (1998) Neuromuscular functioning of athletes and non-athletes in the drop jump. Eur J Appl Physiol 78(5):432-440

24. Höss-Jelten C (2004) Untersuchungen zu den unmittelbaren Wirkungen verschiedener Dehnmethoden auf ausgewählte Kraftparameter. Technische Universität, München

25. Lord SR, Murray SM, Chapman K, Munro B, Tiedemann A (2002) Sit-to-stand performance depends on sensation, speed, balance, and psychological status in addition to strength in older people. J Gerontol Series A Biol Sci Med Sci 57(8):M539-M543

26. Thomas JR, Nelson JK, Thomas KT (1999) A generalized rankorder method for nonparametric analysis of data from exercise science: a tutorial. Res Q Exerc Sport 70(1):11-23

27. Sabapathy NM, Minahan CL, Turner GT, Broadley SA (2011) Comparing endurance- and resistance-exercise training in people with multiple sclerosis: a randomized pilot study. Clin Rehabil 25(1):14-24. doi:10.1177/0269215510375908

28. Corder GW, Foreman DI (2009) Nonparameztric statistics for non-statisticans. A step-by-step approach. Wiley, Hoboken

29. Erdfelder E, Buchner A, Faul F, Brandt M (2003) GPOWER: Teststärkeanalysen leicht gemacht. In: Funke EEJ (ed) Allgemeine Psychologie und deduktivistische Methodologie, 2nd edn. Vandenhoeck \& Ruprecht, Göttingen, pp 148-166

30. Bortz WM (2002) A conceptual framework of frailty: a review. J Gerontol Series A Biol Sci Med Sci 57(5):M283-M288

31. Rubenstein LZ (2006) Falls in older people: epidemiology, risk factors and strategies for prevention. Age Ageing 35(Suppl 2):ii37-ii41. doi:10.1093/ageing/afl084

32. Haas CT, Turbanski S, Kessler K, Schmidtbleicher D (2006) The effects of random whole-body-vibration on motor symptoms in Parkinson's disease. NeuroRehabilitation 21(1):29-36

33. Turbanski S, Haas CT, Schmidtbleicher D, Friedrich A, Duisberg P (2005) Effects of random whole-body vibration on postural control in Parkinson's disease. Res Sports Med 13(3):243-256

34. Schuhfried O, Mittermaier C, Jovanovic T, Pieber K, PaternostroSluga T (2005) Effects of whole-body vibration in patients with multiple sclerosis: a pilot study. Clin Rehabil 19(8):834-842

35. Iwamoto J, Sato Y, Takeda T, Matsumoto H (2012) Whole body vibration exercise improves body balance and walking velocity in postmenopausal osteoporotic women treated with alendronate: Galileo and Alendronate Intervention Trail (GAIT). J Musculoskelet Neuronal Interact 12(3):136-143

36. Runge M, Rehfeld G, Resnicek E (2000) Balance training and exercise in geriatric patients. J Musculoskelet Neuronal Interact 1(1):61-65

37. Foldvari M, Clark M, Laviolette LC, Bernstein MA, Kaliton D, Castaneda C, Pu CT, Hausdorff JM, Fielding RA, Singh MA (2000) Association of muscle power with functional status in community-dwelling elderly women. J Gerontol Series A Biol Sci Med Sci 55(4):M192-M199

38. Skelton DA, Greig CA, Davies JM, Young A (1994) Strength, power and related functional ability of healthy people aged 65-89 years. Age Ageing 23(5):371-377

39. Yamashita F, Iwamoto J, Osugi T, Yamazaki M, Takakuwa M (2012) Chair rising exercise is more effective than one-leg standing exercise in improving dynamic body balance: a randomized controlled trial. J Musculoskelet Neuronal Interact 12(2):74-79

40. Knuttgen HG, Komi PV (2003) Basic considerations for exercise. In: Komi PV (ed) Strength and power in sport, 2nd edn. Blackwell Science Ltd, Oxford, pp 3-10

41. Cuoco A, Callahan DM, Sayers S, Frontera WR, Bean J, Fielding RA (2004) Impact of muscle power and force on gait speed in disabled older men and women. J Gerontol Series A Biol Sci Med Sci 59(11):1200-1206 
42. Puthoff ML, Nielsen DH (2007) Relationships among impairments in lower-extremity strength and power, functional limitations, and disability in older adults. Phys Ther 87(10):1334-1347. doi:10.2522/ptj.20060176

43. Rogan S, Radlinger L, Jöhr C, Nyffenegger C, Stuck N-J, Hilfiker $\mathrm{R}$ [Effect of whole body vibration training on strength and power in an elderly population - a systematic review and meta-analysis]. In: Swiss National Physiotherapy Congress Geneva, 2012

44. Herren K, Radlinger L Risks and side-effects of whole body vibration training. In: World Confederation of Physical Therapy Congress, Amsterdam, 2011

45. Lipsitz LA, Jonsson PV, Kelley MM, Koestner JS (1991) Causes and correlates of recurrent falls in ambulatory frail elderly. J Gerontol 46(4):M114-M122

46. Nevitt MC, Cummings SR, Kidd S, Black D (1989) Risk factors for recurrent nonsyncopal falls. A prospective study. JAMA J Am Med Assoc 261(18):2663-2668

47. Guralnik JM, Ferrucci L, Simonsick EM, Salive ME, Wallace RB (1995) Lower-extremity function in persons over the age of 70 years as a predictor of subsequent disability. N Engl J Med 332(9):556-561. doi:10.1056/NEJM199503023320902
48. Martin BJ, Park HS (1997) Analysis of the tonic vibration reflex: influence of vibration variables on motor unit synchronization and fatigue. Eur J Appl Physiol 75(6):504-511

49. Porter Starr KN, McDonald SR, Bales CW (2014) Obesity and physical frailty in older adults: a scoping review of lifestyle intervention trials. J Am Med Dir Assoc. doi:10.1016/j.jamda.2013.11.008

50. Baumgartner RN, Koehler KM, Gallagher D, Romero L, Heymsfield SB, Ross RR, Garry PJ, Lindeman RD (1998) Epidemiology of sarcopenia among the elderly in New Mexico. Am J Epidemiol 147(8):755-763

51. Forbes GB (1999) Longitudinal changes in adult fat-free mass: influence of body weight. Am J Clin Nutr 70(6):1025-1031

52. Annesi JJ, Vaughn LL (2011) Relationship of exercise volume with change in depression and its association with self-efficacy to control emotional eating in severely obese women. Adv Prev Med 2011:514271. doi:10.4061/2011/514271

53. Luginbuehl H, Lehmann C, Gerber R, Kuhn A, Hilfiker R, Baeyens JP, Radlinger L (2012) Continuous versus intermittent stochastic resonance whole body vibration and its effect on pelvic floor muscle activity. Neurourol Urodyn 31(5):683-687. doi:10. $1002 /$ nau.21251 